\title{
IVD.24 - Recombinant ZIKV envelope proteins for the arbovirosis differential diagnosis by ELISA
}

Juliana Torres de Freitas ${ }^{1 *}$; Paulo de Alvarenga C. Terra ${ }^{1}$; Jorge Luís dos Santos Gonçalves²; Marta Guimarães Cavalcanti³; Mauro Jorge Cabral Castro²; Regina Helena Saramago Peralta ${ }^{1}$; José Mauro Peralta2.

1UFF - Universidade Federal Fluminense;

2UFRJ - Universidade Federal do Rio de Janeiro;

3UFRJ/ HUCFF.

Introduction: The Zika virus is an arbovirus of the Flaviviridae family that causes asymptomatic and symptomatic infections. The clinical diagnosis of arboviroses is difficult in areas with cocirculation of these viruses and, therefore, laboratory confirmation based on host immune response is important, especially for pregnant women. The use of recombinant proteins as antigens aims to improve the sensitivity and specificity of the immunological test.

Objective: The goal of this study is the production of recombinant ZIKV envelope (ENV) protein and the standardization of an ELISA for the arbovirosis differential diagnosis.

Methodology: Expression of the ZIKV partial ENV recombinant protein was performed using a synthetic DNA inserted into plasmid pET28a and expressed in Escherichia coli Rosetta strain. The selected region from ENV protein was the one that showed less homology with ENV Dengue protein. Indirect ELISA tests for IgG detection were standardized using the recENV in the concentration of $0.25 \mu \mathrm{g}$ per well. Forty three sera from individuals living in a mountain community before Zika and Chikungunya epidemics were collected and used as controls. Reactivity threshold was calculated by the mean of the results from control sera plus two standard deviations. The ELISA conditions were: sera dilution 1:100, alkaline phosphataselinked anti-human IgG conjugate dilution of 1:5000 and after 20 minute of incubation reaction was developed using pNPP substrate and reading at $405 \mathrm{~nm}$. Samples from 8 follow-up patients, ZIKV positives by real-time PCR, collected on days 1-2, 5-14 and 20-70 after medical examination were used for analysis.

Results: The recombinant protein was expressed as $30 \mathrm{kDa}$ and express sequence was confirmed by sequencing. In six patients tested in "homemade" IgG Zika ENV assay, the levels of IgG showed a tendency to increase with the infection time but not significantly. On the other hand, using a ZIKV IgM capture Elisa Kit from InBios (Seattle, WA 98109, EUA) in order to compare IgM and IgG levels, we found that specific IgM decreased in all follow-up samples, even the ones that still remained positive.

Conclusion: These results were consistent with $\operatorname{IgG}$ and $\operatorname{IgM}$ antibodies kinetics production. Low levels of IgG in all samples could be explained by the presence in of high levels of IgM. Therefore, even as preliminary results, the use of recombinant ZIKV ENV protein showed to be useful as antigen in immunological methods for detection of antibodies against ZIKV.

Keywords: Zika virus; Recombinant proteins; ELISA 